\title{
Distinct patterns and processes of abundant and rare eukaryotic plankton communities following a reservoir cyanobacterial bloom
}

\author{
Yuanyuan Xue ${ }^{1,2,3} \cdot$ Huihuang Chen ${ }^{1} \cdot$ Jun R. Yang ${ }^{1,2} \cdot$ Min Liu $^{1,2,3} \cdot$ Bangqin Huang $^{3} \cdot$ Jun Yang (1)
}

Received: 13 January 2018 / Revised: 29 April 2018 / Accepted: 1 May 2018 / Published online: 13 June 2018

(c) The Author(s) 2018. This article is published with open access

\begin{abstract}
Plankton communities normally consist of few abundant and many rare species, yet little is known about the ecological role of rare planktonic eukaryotes. Here we used a $18 \mathrm{~S}$ ribosomal DNA sequencing approach to investigate the dynamics of rare planktonic eukaryotes, and to explore the co-occurrence patterns of abundant and rare eukaryotic plankton in a subtropical reservoir following a cyanobacterial bloom event. Our results showed that the bloom event significantly altered the eukaryotic plankton community composition and rare plankton diversity without affecting the diversity of abundant plankton. The similarities of both abundant and rare eukaryotic plankton subcommunities significantly declined with the increase in time-lag, but stronger temporal turnover was observed in rare taxa. Further, species turnover of both subcommunities explained a higher percentage of the community variation than species richness. Both deterministic and stochastic processes significantly influenced eukaryotic plankton community assembly, and the stochastic pattern (e.g., ecological drift) was particularly pronounced for rare taxa. Co-occurrence network analysis revealed that keystone taxa mainly belonged to rare species, which may play fundamental roles in network persistence. Importantly, covariations between rare and non-rare taxa were predominantly positive, implying multispecies cooperation might contribute to the stability and resilience of the microbial community. Overall, these findings expand current understanding of the ecological mechanisms and microbial interactions underlying plankton dynamics in changing aquatic ecosystems.
\end{abstract}

\section{Introduction}

Disturbance can have profound and multiple effects on ecosystems, greatly altering natural community structure [1]. In aquatic systems, cyanobacterial blooms are a common biological disturbance affecting plankton communities [2]. Eukaryotic plankton play an important role

Electronic supplementary material The online version of this article (https://doi.org/10.1038/s41396-018-0159-0) contains supplementary material, which is available to authorized users.

$\triangle$ Jun Yang

jyang@iue.ac.cn

1 Aquatic EcoHealth Group, Key Laboratory of Urban Environment and Health, Institute of Urban Environment, Chinese Academy of Sciences, Xiamen 361021, China

2 University of Chinese Academy of Sciences, Beijing 100049, China

3 College of Environment and Ecology, Xiamen University, Xiamen 361102, China in the trophic web structure [3], and cyanobacterial blooms may change food webs by inhibiting the growth of other phytoplankton, further influencing resource use efficiency in phytoplankton and zooplankton communities $[4,5]$. As important components of food webs, eukaryotic plankton carry out a range of ecological roles, such as primary producers, bacterivores, planktivores, parasites, and saprotrophs $[6,7]$. Thus their responses to environmental fluctuations may directly influence aquatic ecosystem functioning [8]. However, there are significant knowledge gaps about how environmental conditions shape whole eukaryotic plankton communities (including Protists and Fungi) [9]. Reservoirs are one of suitable freshwater ecosystems in which to study the succession of eukaryotic plankton communities, owing to their highly dynamic environment dominated by diverse microorganisms [10].

Eukaryotic plankton are extremely diverse with relatively few abundant species co-existing with a large number of rare species, the latter often referred to as the "rare biosphere" $[11,12]$. Owing to methodical limitations, previous analyses of eukaryotic plankton communities have mainly 
focused on relatively abundant taxa through microscope observation [13]. These abundant taxa contribute to the fluxes of organic matter and biomass production [14], thereby studying their community composition is important for understanding ecological function. Rapid advances in molecular approaches have substantially boosted our knowledge of rare microbial diversity. In particular, highthroughput sequencing technologies have been successfully applied to compare the composition and dynamics of abundant and rare eukaryotic communities in various aquatic ecosystems, including coastal waters and intertidal sediments [15], epipelagic waters [16], and reservoirs [17].

Recent studies have increasingly emphasized the ecological importance of the rare biosphere, because rare taxa can include more metabolically active microorganisms than abundant taxa (as measured by RNA to DNA ratios), and they may be keystone species in regulating the functioning of aquatic environments [18, 19]. The rare microbes have been shown to fulfill essential functions associated with nutrient cycling, and may enhance functionality of the abundant microbes (as reviewed in Jousset et al. [20]). Additionally, as part of the microbial "seed bank", rare taxa can potentially drive ecosystem responses to environmental changes and become dominant under favorable conditions [21], therefore providing a mechanism for community persistence and stability [22]. In aquatic ecosystems, the response of plankton communities to environmental change is mediated by their properties, such as physiological tolerance, dispersal capacity, and taxonomic, and functional diversity [8, 23]. Abundant and rare community assemblages are likely subject to different controlling factors. For example, previous marine studies showed that in surface waters, abundant taxa were primarily influenced by dispersal limitations, while rare taxa were mainly controlled by environmental filtering [16].

Cyanobacterial blooms can have a major impact on the microbial community through both direct (e.g., species interactions) and indirect (e.g., bloom-induced changes in water properties) effects $[2,24]$. While there has been considerable research conducted on the interactions between the abundant planktonic eukaryotes and cyanobacteria $[25,26]$, few studies have investigated how cyanobacterial blooms affect rare planktonic eukaryotes in the water column. A complex network of interrelationships can reveal the intrinsic mechanisms of microbial interactions in response to environmental disturbance [27]. When considering the whole eukaryotic community, the complex cooccurrence networks between interacting microorganisms (e.g., autotrophs, heterotrophs, and parasites; abundant and rare taxa) and the topological features of these networks can be explored. Such networks have been constructed to unveil bacterial interactions in a range of systems, such as river
[28], oil-contaminated soil [29], and marine [30] ecosystems. Until now, however, the co-occurrence patterns of rare and abundant eukaryotes, particularly in reservoir and lake ecosystems, have not been investigated.

Here, for the first time, we investigated the temporal patterns of eukaryotic plankton communities using $18 \mathrm{~S}$ rRNA gene-based high-throughput sequencing and explored the associations between abundant and rare planktonic eukaryotes based on network analysis in a subtropical reservoir. This reservoir system was experiencing a cyanobacterial bloom at the beginning of the sampling period, and we tracked the biotic and abiotic changes through the three months following the bloom event. Our main objectives are to (i) compare the diversity and composition of abundant and rare eukaryotic plankton communities, and their relative contributions to community shift over time; (ii) uncover the co-occurrence patterns of abundant and rare planktonic eukaryotes; (iii) identify the controlling mechanisms and factors that influence the dynamics of the eukaryotic plankton community. Taken together, this research will enhance our understanding of the dynamics of eukaryotic plankton communities in variable environments, with special emphasis on the rare biosphere.

\section{Materials and methods}

\section{Sampling and environmental information}

The study site (Xidong Reservoir, $24^{\circ} 49^{\prime} \mathrm{N}, 118^{\circ} 10^{\prime} \mathrm{E}$ ), and the sampling design have been previously described in Xue et al. [24]. Briefly, water samples $(n=18)$ were collected twice a month from October to December 2014 across three discrete depths: surface $(0.5 \mathrm{~m})$, middle (thermocline or oxycline at $12,14,17,17,20$, and $18 \mathrm{~m}$, for the six sampling dates) and bottom $(25 \mathrm{~m})$ water layers. The cyanobacterial bloom was observed in October, and the reservoir recovered in November based on chlorophyll $a$ concentration and water transparency (Fig. S1). Microscopic inspection showed that this bloom was almost exclusively ( $>80 \%$ of phytoplankton biomass) composed of Microcystis aeruginosa (Fig. S2). A temporal frame work divided into three successional periods is used here: the bloom period in October, post-bloom period 1 in November, and post-bloom period 2 in December. Water samples were divided into two subsamples: one for eukaryotic plankton community analyses and the other for water chemistry. For the eukaryotic plankton, $500 \mathrm{~mL}$ of water was filtered through $0.22 \mu \mathrm{m}$ polycarbonate filters $(47 \mathrm{~mm}$ diameter, Millipore, Billerica, MA, USA). Filters were stored at $-80^{\circ} \mathrm{C}$ until further processing. In total, 17 measured environmental variables in the water were taken 
directly from our previous study [24]. Transparency was determined with a $30 \mathrm{~cm}$ Secchi disk. Chlorophyll $a$ was measured with a PHYTO-PAM Phytoplankton Analyzer (Heinz Walz GmbH, Eichenring, Germany).

\section{DNA extraction, PCR, and Illumina sequencing}

Extraction and purification of plankton DNA from filters were carried out using the FastDNA spin kit for soil (MP Biomedicals, Santa Ana, CA, USA) following the manufacturer's instructions. The V9 region of the eukaryotic small-subunit 18S rRNA gene was amplified using the primer set $1380 \mathrm{~F}$ ( $5^{\prime}$-CCCTGCCHTTTGTACACAC- $\left.3^{\prime}\right)$ and 1510R (5'-CCTTCYGCAGGTTCACCTAC-3') [31]. Each plankton DNA sample was PCR-amplified in triplicate. The $30 \mu \mathrm{L}$ PCR mixture contained $15 \mu \mathrm{L}$ of Phusion High-Fidelity PCR Master Mix (New England Biolabs, Beverly, MA, USA), $0.2 \mu \mathrm{M}$ of each primer, and $10 \mathrm{ng}$ of sample DNA. The PCR reaction conditions were $98^{\circ} \mathrm{C}$ for $1 \mathrm{~min}$, followed by 30 cycles of $10 \mathrm{~s}$ at $98^{\circ} \mathrm{C}, 50^{\circ} \mathrm{C}$ for 30 s, $72{ }^{\circ} \mathrm{C}$ for $30 \mathrm{~s}$, and a final extension at $72{ }^{\circ} \mathrm{C}$ for $5 \mathrm{~min}$. The triplicate PCR products for each sample were pooled in equal quantity for purification and sequenced on the Illumina HiSeq2500 platform (Illumina, Inc., San Diego, CA, USA) using a paired-end $(2 \times 250 \mathrm{bp})$ approach [17].

\section{Bioinformatics}

Paired-end reads from the raw DNA fragments were assembled with FLASH and the mean contig read length was $137 \pm 0.6 \mathrm{bp}$ [32]. For the 18 water samples, we obtained 2,532,735 raw reads, ranging from 127,914 to 155,100 with a mean of 140,707 reads per sample. Sequence processing was performed using the quantitative insights into microbial ecology (QIIME v.1.8.0) pipeline with the following settings: reads with an average Phred score $<25$ were discarded; maximum number of consecutive low-quality base $=3$; minimum of continuous high-quality base $=75 \%$ of total read length; maximum number of ambiguous bases $=0$, last quality score $=3$ [33]. Chimeric sequences were identified by UCHIME and removed before downstream analysis [34]. The UPARSE pipeline was used to pick operational taxonomic units (OTUs) at 97\% similarity level [35]. Subsequently, a representative sequence from each OTU was aligned against the SILVA 123 reference alignment using the RDP classifier [36]. To minimize inclusion of sequencing errors, singletons (OTUs with only one sequence) were eliminated in the whole data set, and then we used a randomly selected subset of 123,090 sequences from each sample to normalize sequencing effort across samples. The final total data set retained 2305 OTUs and 2,215,620 reads at 97\% similarity level.

\section{Definition of abundant and rare taxa}

The definition of abundant and rare taxa depends on the cutoff level of relative abundance, setting 0.1 or $0.01 \%$ as rare OTUs and $1 \%$ as abundant OTUs $[11,15,17]$. These classifications can neglect the intermediate taxa (i.e., relative abundance between 0.1 or 0.01 and $1 \%$ ) and the oscillating taxa (i.e., rare and abundant under different conditions). In this study, we classified all OTUs into six categories following recent studies [12, 37]: always abundant taxa (AAT) with a relative abundance $\geq 1 \%$ in all samples; conditionally abundant taxa (CAT) with a relative abundance $\geq 0.01 \%$ in all samples and $\geq 1 \%$ in some samples; always rare taxa (ART) with a relative abundance $<0.01 \%$ in all samples; conditionally rare taxa (CRT) with a relative abundance $<0.01 \%$ in some samples but never $\geq 1 \%$ in any sample; moderate taxa with a relative abundance between $0.01 \%$ and $1 \%$ in all samples (MT); conditionally rare and abundant taxa (CRAT) with a relative abundance ranging from rare $(<0.01 \%)$ to abundant $(\geq 1 \%)$. Then, for the comparative study of abundant and rare taxa, the abundant taxa (AT) consisted of always abundant and conditionally abundant taxa, and the rare taxa (RT) composed of always rare and conditionally rare taxa. Detailed and general descriptions of abundant and rare data sets are presented in Supplementary Tables S1 and S2.

\section{Ecological inference}

We tentatively performed a basic functional annotation to discriminate OTUs corresponding to pigmented and nonpigmented taxa. Ecological inference was based on the literature describing the presence or absence of chloroplasts in microbial eukaryotic groups [3, 38, 39]. Pigmented groups comprised both obligate phototrophs (e.g., Chlorophyta and Diatomea) and mixotrophs (e.g., Chrysophyceae, Cryptophyceae, and Dinophyceae). OTUs distributed within groups of free-living heterotrophs (e.g., Ciliophora, Cercozoa, and osmotrophic Fungi) and parasites (Apicomplexa, Chytridiomycota, Perkinsidae, Peronosporomycetes, and Pirsonia) were reliably assigned to non-pigmented taxa. When OTUs could not be precisely identified (i.e., to at least the genus level), they were assigned to "unknown" ecological roles (e.g., unidentified Alveolata).

\section{Statistical analyses}

\section{Alpha-diversity analysis}

Alpha-diversity indices were computed using the diversity function in the "vegan" R package. Spatiotemporal effects on alpha-diversity were examined with two-way analysis of variance (two-way ANOVA) using SPSS Statistics for 
Windows v.22.0 (IBM Corp., Armonk, NY, USA). To estimate sampling effort, we fitted our data to a truncated Preston log-normal distribution using the prestonfit and prestondistr functions of the "vegan" $\mathrm{R}$ package [40].

\section{Beta-diversity analysis}

Beta-diversity was measured using Bray-Curtis dissimilarity [41]. The beta-diversity values of different subcommunities were partitioned into two components, the balanced variation (richness) and abundance gradient (turnover), using the bray.part function of the "betapart" $\mathrm{R}$ package [29]. To reveal temporal patterns in eukaryotic plankton communities, a time-lag analysis was used to quantify the Bray-Curtis dissimilarity between each pair of samples, and the time difference (lag) was then plotted against the dissimilarity [42]. Eukaryotic plankton community composition was visualized using non-metric multidimensional scaling (NMDS) based on Bray-Curtis dissimilarities. Analysis of similarity (ANOSIM) was used to investigate differences in eukaryotic plankton communities between groups. The contribution of each species (i.e., OTU) to community dissimilarity over time was calculated using similarity percentage (SIMPER) analysis. The NMDS, ANOSIM, and SIMPER analyses were performed using PRIMER v.7.0 and PAST v.2.12. Finally, to further explain the patterns of beta-diversity, we calculated Levin's niche breadth index separately for the abundant and rare taxa [43].

\section{Indicator OTUs associated with environmental changes}

We determined which OTUs could explain the time effect in diversity by identifying indicator species in the "indicspecies" $R$ package [44]. This analysis calculates an indicator value that measures the association between OTUs and groups of samples and then identifies the group corresponding to the highest association value. For this study, we compared samples across three periods (cyanobacterial bloom period, post-bloom period 1, and post-bloom period 2) to determine temporal indicators based on an indicator value $>0.7$ and $P$-value $<0.05$ assessed after 999 permutation tests.

\section{Network analysis}

To reduce the complexity of the data sets, OTUs present in more than six samples with more than 20 sequences were retained for the construction of networks. A total of 1058 OTUs were used for the network analyses. Subsequently, all possible pairwise Spearman's rank correlations $(r)$ between those OTUs were calculated within the "picante" $\mathrm{R}$ package. Only robust $(r>0.8$ or $r<-0.8)$ and statistically significant $(P$-value $<0.01)$ correlations were incorporated into network analyses [28]. Network visualization and modular analysis were made with Gephi version 0.8.2. Node-level topological properties (i.e., degree, betweenness, closeness, and eigenvector) were further calculated in the "igraph" $\mathrm{R}$ package. Statistical differences in measured node-level attributes across different taxa were determined using nonparametric Mann-Whitney $U$ test. Nodes with high degree $(>100)$ and low betweenness centrality values $(<5000)$ are recognized as keystone species in co-occurrence networks [45]. Hubs (i.e., highly linked species within their own module) and connectors linking different modules in co-occurrence network were identified based on their connectivity as described previously [46]. Meanwhile, 1000 Erdös-Réyni random networks, which had the identical number of nodes and edges as the real networks, were generated in the "igraph" R package, with each edge having the same probability of being assigned to any node [47]. Topology characteristics of both real and random networks were calculated and compared, including modularity, clustering coefficient, and average path length.

\section{Relationships between community composition and environment variables}

We implemented the Mantel test using the "vegan" package in R to select significant environmental factors correlated with the variations of eukaryotic plankton communities. Prior to the analysis, the normality of the physicochemical variables was checked using the Shapiro-Wilk test, and log $(x+1)$ transformed, with the exception of $\mathrm{pH}$, to improve normality and homoscedasticity. Additionally, Spearman correlations were calculated to discern the relationship between the OTUs of each major module and environmental variables in the "picante" $\mathrm{R}$ package. Only robust (Spearman's $r>0.8$ or $r<-0.8$ ) and statistically significant $(P$-value $<0.01)$ correlations were considered in this study.

\section{Neutral community model}

A neutral community model was used to determine the potential contribution of neutral processes to eukaryotic plankton community assembly by predicting the relationship between OTUs occurrence frequency and their relative abundance [48]. This model predicts that rare taxa will be lost with time due to ecological drift, while abundant taxa are more likely to be dispersed by chance and thus present in more samples. In this model, $R^{2}$ and $\mathrm{Nm}$ values indicate the fit to the neutral model and metacommunity size times immigration, respectively.

\section{Accession numbers}

All raw sequences data from this study have been submitted to the NCBI Sequence Read Archive (SRA) database under 
the BioProject number PRJNA348137 and the accession number SRP091963.

\section{Results}

\section{General patterns of species richness and alpha- diversity}

Overall, the sequencing of 18S rRNA genes yielded $2,215,620$ high-quality sequences and 2305 OTUs at $97 \%$ similarity level. The total number of OTUs (2305) in all samples was roughly equivalent to the richness estimated by Chao $1(2372 \pm 15)$ and ACE $(2371 \pm 24)$ (Table S2). The rarefaction curves almost approached saturation for the total and three separate periods (Fig. S3a). Further, we fitted our community data to the truncated Preston log-normal model (Fig. S3b), and estimated that our sampling found $87-88 \%$ of the OTUs in the studied reservoir. These results indicated that the majority of the eukaryotic plankton diversity had been recovered by the deep sequencing.

In the whole data set, 44 OTUs $(1.91 \%)$ representing $73.04 \%$ of all sequences were abundant and persistent across all periods, whereas 2167 OTUs $(94.01 \%)$ contributing $13.84 \%$ of all sequences were affiliated to rare taxa (Table S2). The non-pigmented taxa (mainly Ciliophora, Fungi, and Cercozoa) dominated the OTU richness, together representing $40.5 \%$ of the total OTUs (Table S3). Pigmented groups, dominated by Chlorophyta (117 OTUs), Chrysophyceae (99 OTUs), and Cryptophyceae (94 OTUs), accounted for only $20.7 \%$ of all OTUs.

Alpha-diversity of all and abundant taxa showed a similar pattern, and did not differ over depth and time (Table 1). However, rare taxa exhibited a significant temporal pattern with an increase in Shannon-Wiener diversity, Simpson diversity, and Pielou's evenness from bloom to post-bloom 1, followed by a loss of these indices during post-bloom 2 (Table 1 and Fig. S4). Venn diagram analysis indicated that most of the OTUs were shared among three periods, and all of the period-specific OTUs belonged to rare taxa (Fig. 1b). Additionally, we found high variability in the number of strict indicator OTUs over time (ranging from 41 during bloom period, 3 during post-bloom period 1 to 87 during post-bloom period 2). The taxonomic compositions of strict indicators were significantly different among three different periods (Table S4).

\section{Temporal dynamics in microbial community composition}

Generally, the time-lag regression analysis had significant positive slopes, indicating both microbial plankton communities and environmental conditions were undergoing a
Table 1 Two-way ANOVA showing the effects of time and depth on the alpha-diversity of eukaryotic plankton

\begin{tabular}{|c|c|c|c|c|c|c|}
\hline & \multicolumn{2}{|l|}{ All } & \multicolumn{2}{|c|}{ Abundant } & \multicolumn{2}{|l|}{ Rare } \\
\hline & $F$ & $P$ & $F$ & $P$ & $F$ & $P$ \\
\hline \multicolumn{7}{|l|}{ Time } \\
\hline Richness & 1.789 & 0.222 & & & 1.792 & 0.221 \\
\hline $\mathrm{ACE}$ & 1.674 & 0.241 & & & 1.678 & 0.240 \\
\hline Chao 1 & 1.646 & 0.246 & & & 1.658 & 0.244 \\
\hline Shannon-Wiener & 1.264 & 0.328 & 1.025 & 0.397 & 11.561 & 0.003 \\
\hline Simpson & 1.373 & 0.302 & 1.382 & 0.300 & 9.422 & 0.006 \\
\hline Pielou's evenness & 1.333 & 0.311 & 1.025 & 0.397 & 20.786 & 0.000 \\
\hline \multicolumn{7}{|l|}{ Depth } \\
\hline Richness & 1.356 & 0.306 & & & 1.353 & 0.306 \\
\hline $\mathrm{ACE}$ & 1.166 & 0.354 & & & 1.170 & 0.354 \\
\hline Chao 1 & 1.378 & 0.301 & & & 1.384 & 0.299 \\
\hline Shannon-Wiener & 1.419 & 0.291 & 1.366 & 0.303 & 0.571 & 0.584 \\
\hline Simpson & 1.806 & 0.219 & 1.492 & 0.276 & 0.142 & 0.870 \\
\hline Pielou's evenness & 1.437 & 0.287 & 1.366 & 0.303 & 0.016 & 0.984 \\
\hline \multicolumn{7}{|l|}{ Time $\times$ Depth } \\
\hline Richness & 1.814 & 0.210 & & & 1.802 & 0.213 \\
\hline $\mathrm{ACE}$ & 1.470 & 0.289 & & & 1.452 & 0.294 \\
\hline Chao 1 & 1.511 & 0.278 & & & 1.508 & 0.279 \\
\hline Shannon-Wiener & 1.207 & 0.372 & 0.857 & 0.525 & 0.357 & 0.833 \\
\hline Simpson & 0.865 & 0.520 & 0.686 & 0.619 & 0.567 & 0.693 \\
\hline Pielou's evenness & 1.125 & 0.403 & 0.857 & 0.525 & 0.188 & 0.939 \\
\hline
\end{tabular}

Note that abundant OTUs were persistent across all samples, and the $F$ and $P$ values of richness, ACE, and Chao 1 indices cannot be calculated

Time indicates three successional periods, including bloom, postbloom 1, and post-bloom 2 periods

Depth denotes surface, middle, and bottom water layers

All, whole eukaryotic plankton; Abundant, abundant eukaryotic plankton; Rare, rare eukaryotic plankton

Bold font means the significance at $P<0.05$ level

directional change (Fig. 2a-c and Fig. S5). However, the slope for the rare taxa was significantly steeper than those in the all and abundant taxa. Beta-diversity partitioning further revealed that species replacement (turnover), rather than species richness, accounted for the majority of the betadiversity, and drove the shift in community composition over time (Fig. 2d-f and Fig. S6).

Our results revealed that eukaryotic plankton communities clustered strongly by time rather than depth, and three groups of samples were clearly defined: bloom, post-bloom 1, and post-bloom 2 (Fig. 1a). The Mantel test results also demonstrated that eukaryotic plankton communities were mainly governed by temporal factors (Table 2). Rare taxa showed a striking separation compared with the all and abundant taxa, confirmed by the comparison of between- 

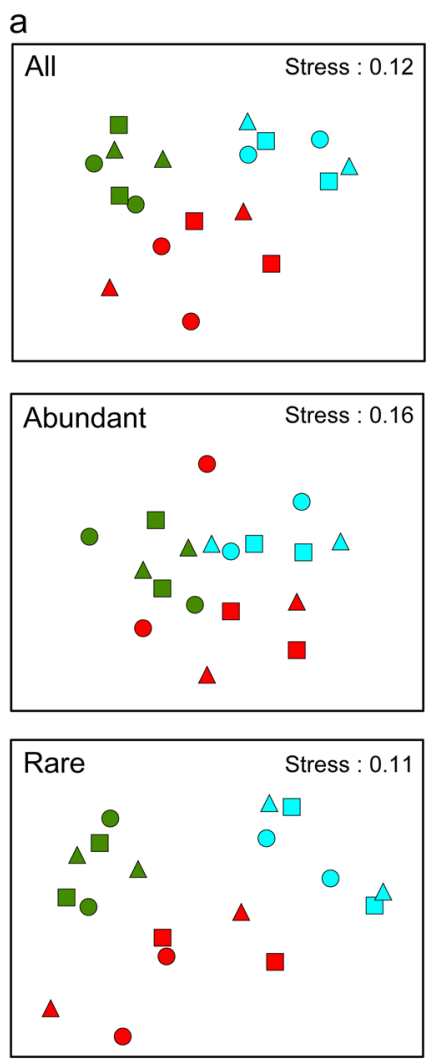

$\square$ Bloom $\square$ Post-bloom $1 \square$ Post-bloom 2 - Surface $\boldsymbol{\square}$ Middle $\boldsymbol{\Delta}$ Bottom b
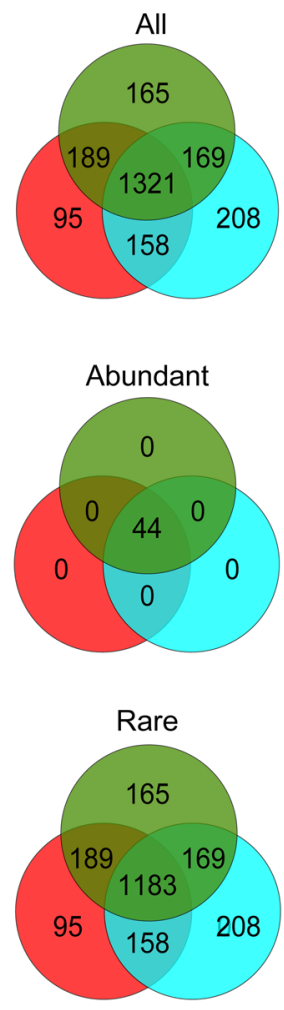

Bloom Post-bloom 1 Post-bloom 2
C

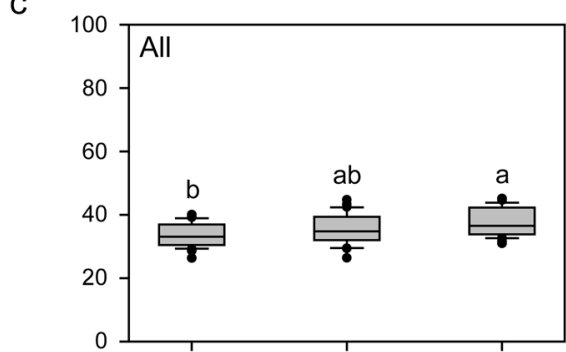

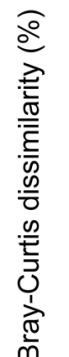
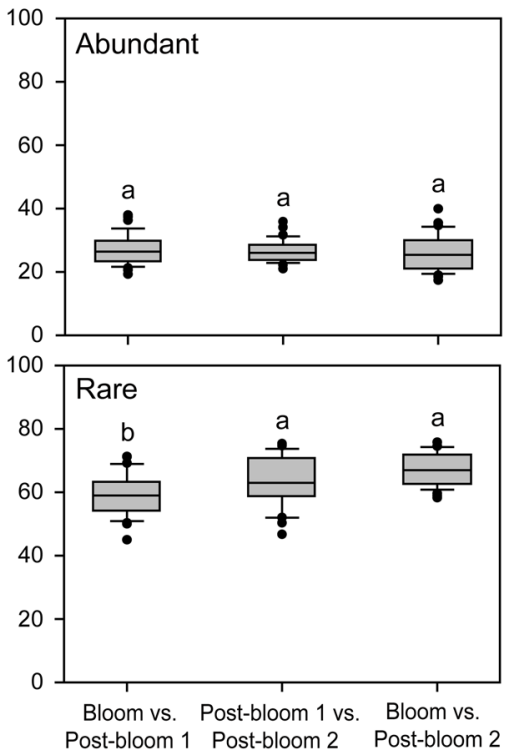

Fig. 1 Community structuring of eukaryotic plankton across bloom event. a Non-metric multidimensional scaling (NMDS) ordination of eukaryotic plankton communities based on Bray-Curtis distances. b Venn diagram showing the numbers of unique and shared OTUs between three different periods. c The pairwise Bray-Curtis dissimilarity of eukaryotic plankton communities between different periods. The top and bottom boundaries of each box indicate the 75th and 25th quartile values, respectively, and lines within each box represent the median values $(n=$ 36). Different letters above bars indicate a significant difference at the $P<0.05$ level according to nonparametric Mann-Whitney $U$ test. All, whole eukaryotic plankton communities; Abundant, abundant plankton subcommunities; Rare, rare plankton subcommunities

group distances among different periods and the results of ANOSIM analyses (Fig. 1c and Table 3). Further, abundant taxa exhibited greater niche breadth values than rare taxa among three periods (Fig. S7).

A greater number of taxonomic groups were present among rare planktonic eukaryotes than abundant ones (Fig. S8). The abundant taxa entailed only two major contributors to the community rearrangements, including Arthropoda (6.76\%), for which the relative abundance of sequences declined over time, and Cryptophyceae (2.72\%) characterized by a marked increase from bloom to postbloom periods (Fig. S8c). By contrast, a large number of rare OTUs predominantly contributed to the dissimilarity in community composition. Altogether, they explained $65.76 \%$ of the total dissimilarity over time (Table S1). The main contributors to the rearrangement were unclassified eukaryotic groups (14.76\%), Ciliophora (7.02\%), Chrysophyceae (4.64\%), Cryptophyceae (3.16\%), which had the lowest abundance in post-bloom period 1, Chlorophyta $(3.03 \%)$, Chytridiomycota $(2.22 \%)$, which were particularly abundant during post-bloom period 2, and Cercozoa (3.11\%), which were abundant during the bloom period (Fig. S8d).

\section{Co-occurrence networks of different subcommunities}

A metacommunity co-occurrence network was built based on correlation relationships (Fig. 3a). The resulting network consisted of 791 nodes linked by 9628 edges (Table S5), with a much higher number of strong positive correlations observed than negative ones (Fig. 3a). The network obtained exhibited scale-free characteristics (power-law: $R^{2}$ $=0.956$, Fig. S9), indicating that the network structure was non-random. The observed modularity, average clustering coefficient and average path length were all greater than those of their respective Erdös-Réyni random networks, suggesting the network had "small-world" properties and modular structure (Table S5). Non-rare taxa (abundant, moderate, and conditionally rare and abundant taxa) 


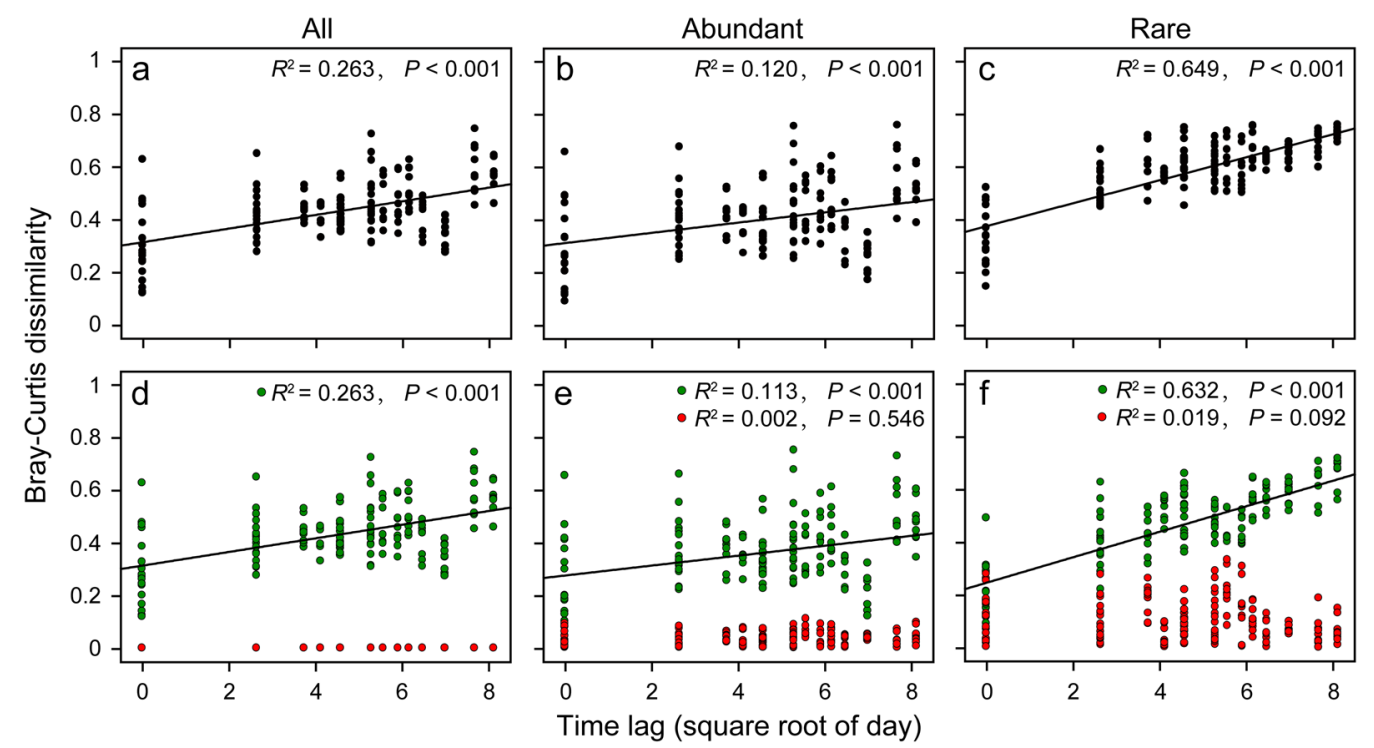

Fig. 2 Time-lag regression analysis of total beta-diversity (a-c), and the turnover (green dots) and richness (red dots) components of beta-diversity (d-f) for all, abundant and rare eukaryotic plankton communities

Table 2 Spearman's correlations of the eukaryotic plankton community with environmental factors based on Mantel tests

\begin{tabular}{|c|c|c|c|c|}
\hline Environmental factors & All & Abundant & Rare & $\begin{array}{l}\text { Co-occurring } \\
\text { network }\end{array}$ \\
\hline Time & $\mathbf{0 . 3 3 4}$ & 0.222 & 0.652 & 0.334 \\
\hline Depth & -0.037 & -0.015 & -0.120 & -0.036 \\
\hline Temperature & 0.215 & 0.211 & 0.235 & 0.220 \\
\hline Electrical conductivity & -0.121 & -0.120 & -0.106 & -0.128 \\
\hline Turbidity & -0.094 & -0.094 & -0.062 & -0.089 \\
\hline $\mathrm{pH}$ & 0.100 & 0.070 & 0.175 & 0.111 \\
\hline $\begin{array}{l}\text { Oxidation reduction } \\
\text { potential }\end{array}$ & -0.132 & -0.139 & -0.027 & -0.141 \\
\hline Dissolved oxygen & 0.075 & 0.071 & -0.039 & 0.069 \\
\hline Total carbon & 0.283 & 0.202 & 0.452 & 0.286 \\
\hline Total organic carbon & 0.226 & 0.193 & 0.293 & 0.233 \\
\hline Total nitrogen & -0.119 & -0.121 & -0.055 & -0.130 \\
\hline Ammonium nitrogen & -0.039 & -0.049 & 0.014 & -0.051 \\
\hline Nitrate nitrogen & 0.100 & 0.110 & 0.042 & 0.098 \\
\hline Nitrite nitrogen & 0.206 & 0.167 & 0.248 & 0.208 \\
\hline Total phosphorus & -0.011 & -0.018 & 0.035 & -0.032 \\
\hline Phosphate phosphorus & -0.105 & -0.073 & -0.156 & -0.121 \\
\hline $\begin{array}{l}\text { Total nitrogen and } \\
\text { total phosphorus ratio }\end{array}$ & 0.148 & 0.117 & 0.224 & 0.136 \\
\hline Chlorophyll $a$ & 0.179 & 0.130 & 0.311 & 0.184 \\
\hline
\end{tabular}

Time indicates three successional periods, including bloom, postbloom 1 , and post-bloom 2 periods

Depth denotes surface, middle, and bottom water layers

All, whole eukaryotic plankton communities; Abundant, abundant plankton subcommunities; Rare, rare plankton subcommunities

The significances are tested based on 999 permutations, and bold values indicate $P<0.05$ frequently interacted more with rare taxa than with themselves (Fig. 3a).

We compared unique node-level topological features of four subcommunities. Both the degree and closeness centrality values of conditionally rare and abundant OTUs were highest among four subcommunities (Fig. 3b,e). Significantly lower betweenness centrality values were found for rare OTUs than for moderate taxa (Fig. 3c). However, eigenvector centrality values showed no significant differences between the four subcommunities (Fig. 3d). Keystone species, which play key roles in maintaining the structure and function of microbial communities, usually have high degree $(>100)$ and low betweenness centrality values $(<5000)$ in co-occurrence networks. Based on this criterion, a total of 17 OTUs were defined as keystone species, including Dinophyceae (1 OTU), Chlorophyta (2 OTUs), Cryptophyceae (2 OTUs), Cercozoa (2 OTUs), Chrysophyceae (3 OTUs), unidentified Stramenopiles (4 OTUs), and unclassified eukaryotic groups (3 OTUs), and all but two of these keystone species belonged to rare taxa (Table S6).

\section{Modular structure of the co-occurrence network}

The entire network was clearly parsed into 6 major modules, of which modules I and II respectively accounted for 30.47 and $18.96 \%$ of the whole network (Fig. 4). Ternary plot analysis indicated that most modules were specific (relatively more abundant) to a particular period (Fig. 5). For example, most of the OTUs from the largest module I had higher relative abundances in the bloom period than post-bloom periods, whereas the majority of the OTUs from 
Table 3 Analysis of similarity (ANOSIM) statistics testing differences of eukaryotic plankton community groupings at spatiotemporal scales

\begin{tabular}{|c|c|c|c|c|c|c|}
\hline \multirow[t]{2}{*}{ Grouping by } & \multicolumn{2}{|l|}{ All } & \multicolumn{2}{|c|}{ Abundant } & \multicolumn{2}{|l|}{ Rare } \\
\hline & $R$ & $P$ & $R$ & $P$ & $R$ & $P$ \\
\hline Global time (month) & 0.757 & 0.001 & 0.568 & 0.001 & 0.825 & 0.001 \\
\hline Bloom vs. Post-bloom 1 & 0.583 & 0.002 & 0.454 & 0.002 & 0.739 & 0.002 \\
\hline Post-bloom 1 vs. Post-bloom 2 & 0.656 & 0.002 & 0.485 & 0.002 & 0.761 & 0.002 \\
\hline Bloom vs. Post-bloom 2 & 0.989 & 0.002 & 0.713 & 0.002 & 0.996 & 0.002 \\
\hline Global space (depth) & -0.129 & 0.961 & -0.115 & 0.944 & -0.152 & 0.991 \\
\hline Surface vs. Middle & -0.087 & 0.729 & -0.017 & 0.506 & -0.144 & 0.903 \\
\hline Middle vs. Bottom & -0.172 & 0.972 & -0.213 & 0.996 & -0.159 & 0.937 \\
\hline Surface vs. Bottom & -0.120 & 0.851 & -0.094 & 0.790 & -0.152 & 0.939 \\
\hline
\end{tabular}

An R-statistic less than 0 represents complete random grouping

All, whole eukaryotic plankton communities; Abundant, abundant plankton subcommunities; Rare, rare plankton subcommunities

Bold font indicates significant value $(P<0.01)$ modules II, III, and VI had higher relative abundances in post-bloom period 2 than the other two periods. Importantly, taxonomic relatedness played a key role in determining the modular structure (Fig. 4a vs. Fig. 4b). The 6 major modules composed of substantial rare OTUs and few non-rare OTUs (abundant, moderate, and conditionally rare and abundant OTUs) were primarily occupied by Chrysophyceae, Cryptophyceae, Dinophyceae, Chlorophyta, Ciliophora, Cercozoa and Fungi (Fig. S10). Based on the values of connectivity, the co-occurrence network had 6 module hubs that belonged to rare taxa, and 12 OTUs were classified as connectors, including 2 abundant OTUs, 3 moderate OTUs, and 7 rare OTUs (Fig. S11). These module hubs and connectors primarily came from module I, IV, V, and VI (Table S7).

\section{Factors related to variation of the eukaryotic plankton community}

The Mantel test results indicated that the changes of all, rare and network communities were correlated with temperature, total carbon (TC), total organic carbon (TOC), and nitrite nitrogen $\left(\mathrm{NO}_{2}-\mathrm{N}\right)$ (Table 2). In addition, $\mathrm{pH}$, total nitrogen and total phosphorus ratio (TN:TP), and chlorophyll $a(\mathrm{Chl}-a)$ were also significantly related to the variation of rare community composition. In contrast to rare taxa, only temperature and TC were significantly correlated with the abundant taxa. More interestingly, the neutral community model successfully explained a large fraction of variation in both all $\left(R^{2}=0.895\right)$ and rare $\left(R^{2}=0.879\right)$ plankton communities (Fig. S12).

To investigate the modules' responses to environmental conditions, significant edge numbers between environmental factors and OTUs from each module were calculated (Spearman's $r>0.8$ or $r<-0.8$, and $P$-value $<0.01$ ).
Among environmental factors, we found that temperature, TC, TOC, $\mathrm{NO}_{2}-\mathrm{N}$, and Chl- $a$ were frequent drivers of network connections (Fig. S13).

\section{Discussion}

Eukaryotic plankton in freshwater ecosystems are considered to be one of the indicators of environmental change and ecosystem state owing to their fast and strong responses to environmental disturbances [2, 23]. We found that abundant and rare eukaryotic plankton communities exhibited different and complex responses to environmental changes. Therefore, elucidating the mechanisms of abundant and rare eukaryotic succession under changing environments is crucial for understanding the process of ecosystem recovery from disturbance events (e.g., cyanobacterial blooms).

\section{Dynamics of abundant and rare eukaryotic plankton communities}

Our results showed that the effect of the cyanobacterial bloom was detectable by its influence on the composition of the eukaryotic plankton community (Fig. 1). The similarity between samples significantly decreased with the increase in time-lag, illustrating that eukaryotic plankton communities were not resilient and had reached an alternative state (Fig. 2a-c). Usually, resilience is defined as the degree to which a system returns to the original state after a perturbation [49]. However, resilience can also be described as the recovery process to an alternative stable state following a disturbance [2]. More importantly, our results indicated that different taxa did not respond equally to the cyanobacterial bloom. Rare taxa showed greater differences in 
Fig. 3 Properties of the correlation-based network. a The networks analysis showing the intra-associations within each subcommunity and interassociations between different subcommunities. A connection stands for a strong (Spearman's $r>0.8$ or $r<-0.8)$ and significant $(P$-value $<0.01)$ correlation. The size of each node is proportional to the number of connections (i.e., degree). Numbers outside and inside parentheses represent positive edge numbers and negative edge numbers, respectively. b-e Comparison of node-level topological features among four different subcommunities. The top and bottom boundaries of each box indicate the 75th and 25th quartile values, respectively, and lines within each box represent the median values. Different letters indicate the significant level at $P<0.05$ level determined by nonparametric Mann-Whitney $U$ test. AT abundant taxa, RT rare taxa, MT moderate taxa, CRAT conditionally rare and abundant taxa a

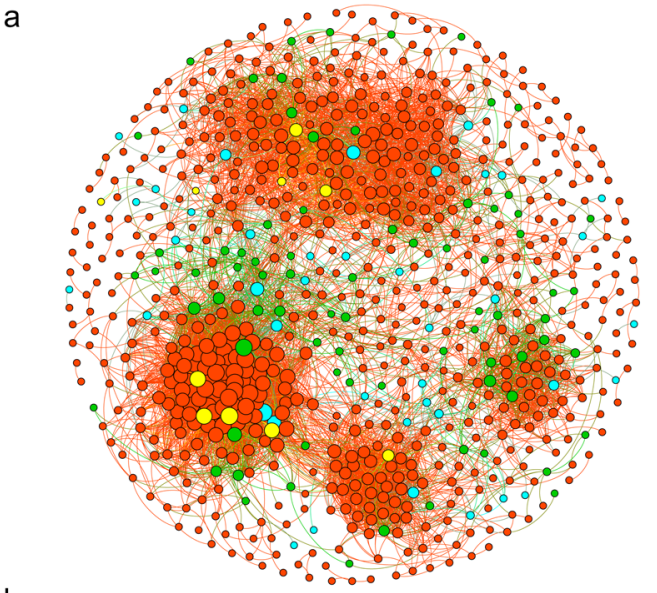

AT $(5.18 \%)$

RT $(83.82 \%)$

MT $(9.74 \%)$

O CRAT $(1.26 \%)$

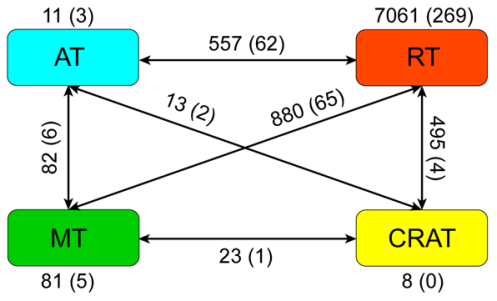

b

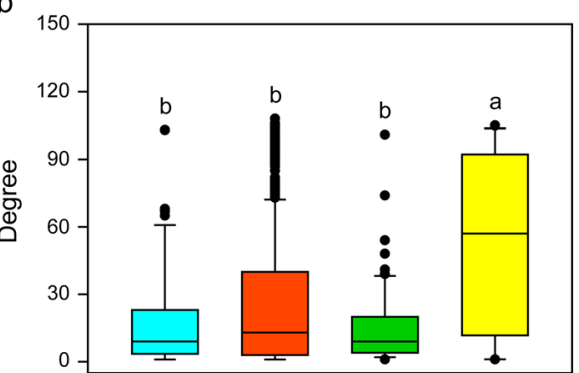

C

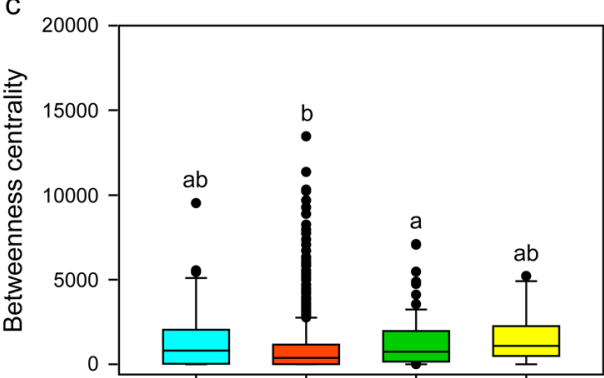

d

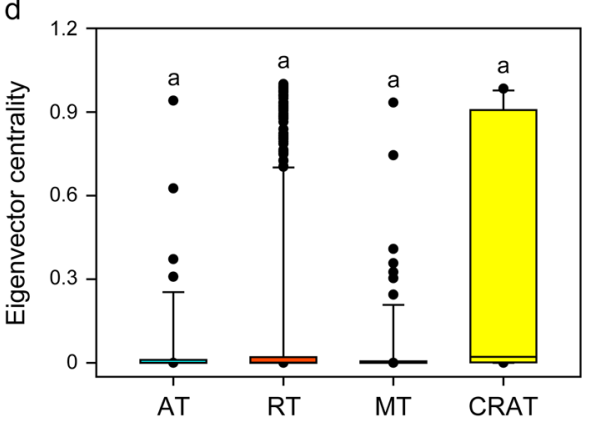

both diversity and community composition between three periods than abundant taxa (Tables 1 and 3), and the temporal turnover of rare taxa $\left(R^{2}=0.649, P<0.001\right)$ was significantly higher than that of the abundant ones $\left(R^{2}=\right.$ $0.120, P<0.001$ ) (Fig. 2b,c). These results indicated that the stable predominance of a few highly abundant taxa is contrasted by a highly dynamic turnover of rare species, as previously observed in Austrian lakes [50].

We found that the cyanobacterial bloom event significantly altered the eukaryotic plankton community composition without affecting overall diversity. This may reflect the fact that microorganisms usually have a high population density, and more individuals with versatile physiology contributing to the high resistance in biodiversity [51]. The rare subcommunities had $\sim 50$ times higher richness than the abundant subcommunities, supporting the idea that rare microbes are important contributors to microbial diversity [19]. Meanwhile, the diversity indices of rare subcommunities exhibited temporal differences, but the abundant subcommunities had no significant difference among three periods. This may be due to the immigration and emigration of rare species and the recovery of dormant taxa [29]. Due to their high diversity, rare populations could increase the functional redundancy of the community [52], further providing biological buffering capacity to withstand environmental changes [53].

\section{Controlling mechanisms and factors shaping the eukaryotic plankton community}

A central challenge in ecology is to quantify the relative roles of deterministic and stochastic processes that shape the assembly of microbial communities [54]. Niche theory holds that microbial communities are shaped by deterministic processes (e.g., habitat heterogeneity or species sorting) owing to different habitat preferences and fitness of 
Fig. 4 The co-occurrence patterns among OTUs revealed by network analysis. The nodes were colored according to different types of modularity classes (a) and supergroups (b), respectively. A connection stands for a strong (Spearman's $r>0.8$ or $r<-0.8)$ and significant $(P$-value $<0.01)$ correlation. The size of each node is proportional to the number of connections (i.e., degree). Major modules have more than 40 nodes. Other modules include all small modules $(n=26)$ with nodes $<18$ per module

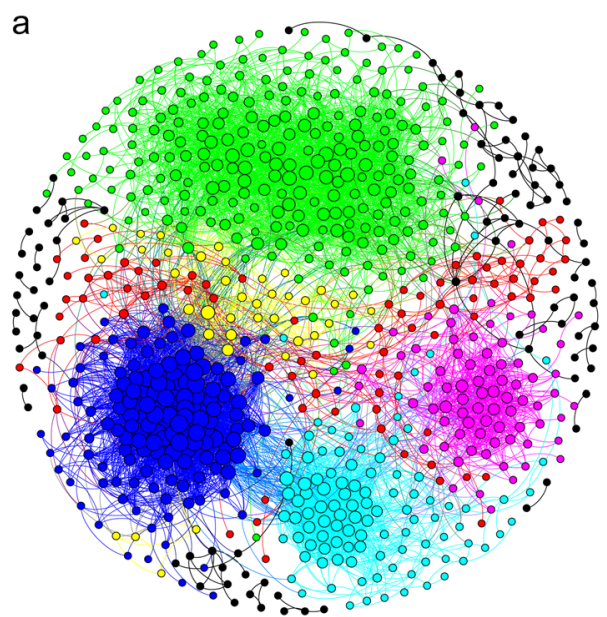

Module I $(30.47 \%) \bullet$ Module II (18.96\%) O Module III (13.15\%)
- Module IV (10.37\%) O Module V $(9.86 \%)$ O Module VI $(5.18 \%)$
Other modules $(12.01 \%)$

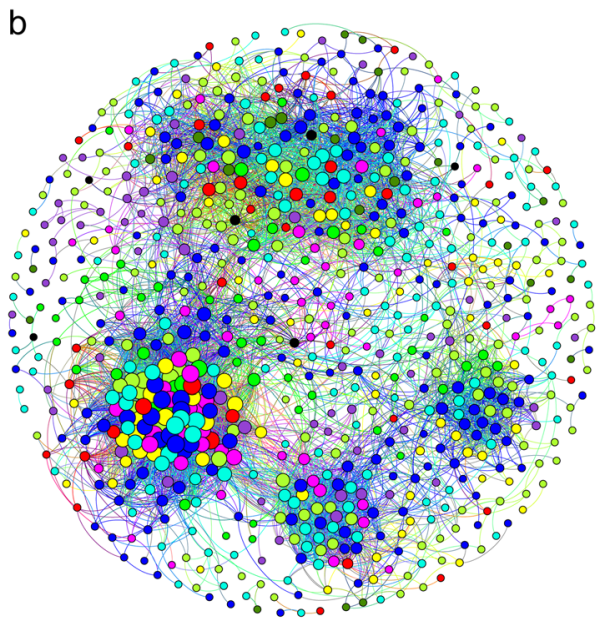

Alveolata (18.96\%) Amoebozoa (0.76\%) Animalia $(7.71 \%)$ Archaeplastida (6.45\%) Excavata (2.78\%) Rhizaria (4.93\%) - Incertae sedis $(7.59 \%)$ Opisthokonta $(9.36 \%)$ - Stramenopiles (23.26\%) O Unclassified (18.20\%)
Fig. 5 Ternary plots showing relative abundance of OTUs from modules I-VI in the three different periods. Each circle represents one individual OTU. For each OTU, abundance was averaged over all samples at each period
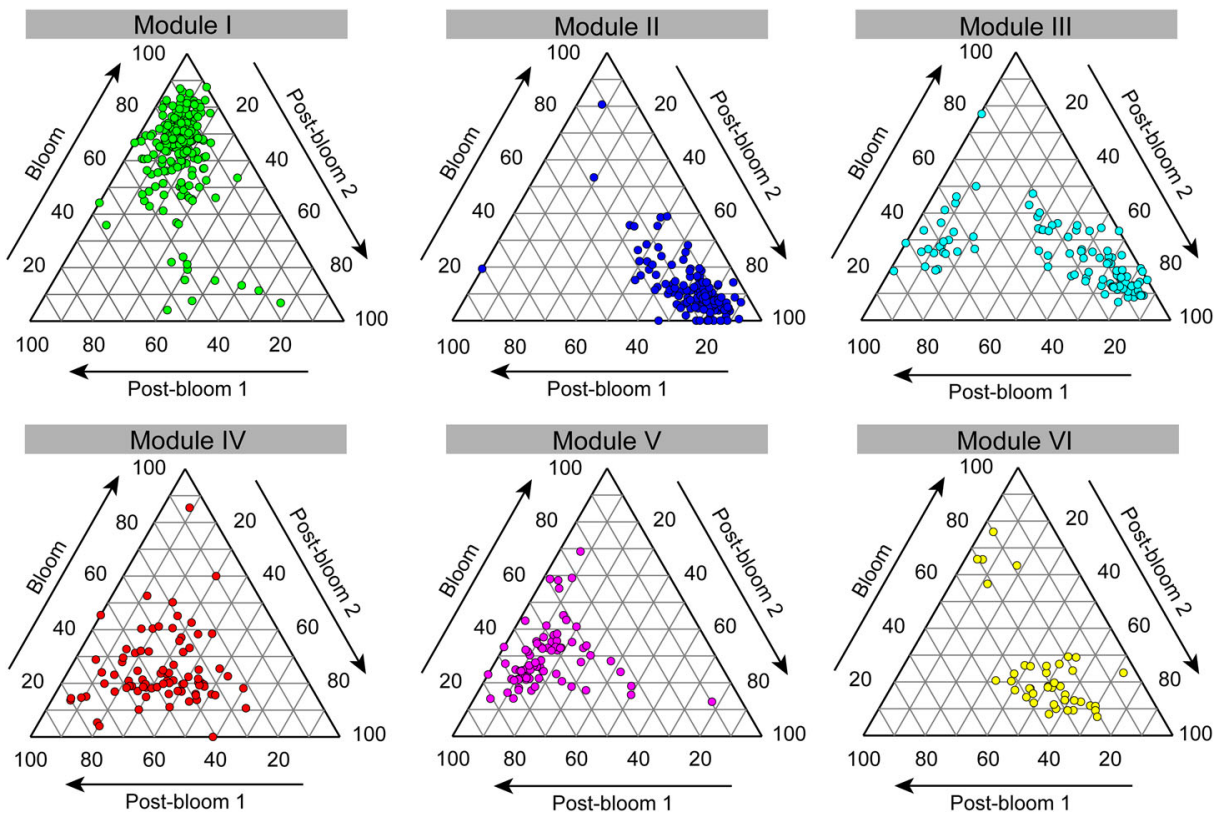

species, whereas according to neutral theory, microbial communities are shaped by random fluctuations in species abundance (birth and death) and limited dispersal [55]. Both deterministic and stochastic processes can act concurrently to regulate the assembly of microbial communities [12]. In this study, water temperature, TC, TOC, $\mathrm{NO}_{2}-\mathrm{N}$ and TN:TP were significantly related to the temporal variability of eukaryotic community composition (Table 2 ). These relationships have also been reported in previous studies of eukaryotic plankton communities from both a shallow lake and a subtropical river $[25,56]$. As expected, rare taxa were more sensitive to environmental filtering compared with abundant taxa. It is possible that abundant taxa competitively utilize a broad array of resources and are well adapted to a particular ecosystem through active growth [57], whereas rare taxa have less competition capability and intrinsically low growth rates [58], thereby being restricted to fewer samples. This suggests that environmentallyinduced species sorting has a strong impact on the composition of microbial communities [59]. Furthermore, abundant taxa had wider temporal niche breadths than rare ones, and a significant positive abundance-occurrence relationship was observed (Fig. S7). The analysis of abundance-occurrence can be a highly valuable approach 
for distinguishing where shifts in microbial communities occur across environmental gradients. Our samples spanned contrasting environmental conditions, where continuous environmental change resulted in the existence of multiple niches. A total of 131 strict indicator OTUs were found across three different periods, and the majority of strict specialists were affiliated to rare taxa (Table S4). This feature suggests that the occurrence and disappearance of cyanobacterial blooms can induce environmental heterogeneity, with local habitat conditions selecting for specific taxonomic groups.

We also found that stochastic processes may play an important role in eukaryotic plankton community assembly. In our study, rare taxa gave a good fit to the neutral model, and over $80 \%$ of community turnover for rare taxa was well explained by stochastic processes (Fig. S12). By investigating the distribution of abundant and rare estuarine fish communities over 21 years, Magurran and Henderson [60] found that abundant species associated with estuarine habitats were log normally distributed, while rare species had different habitat requirements and followed a log series distribution. The stochastic pattern was particularly pronounced for the rare taxa, and so overall the plankton data set was a good fit to the neutral model $\left(R^{2}=0.895\right.$, Fig. S12). Moreover, the proportion of turnover partitioning in the beta-diversity for rare taxa was higher than that of abundant ones (Fig. S6), suggesting that rare taxa with a low abundance are more likely to be lost due to ecological drift (i.e., the stochastic loss and replacement of individuals) [61]. Overall, both deterministic and stochastic processes appear to significantly influence the temporal dynamics of eukaryotic plankton community composition. Recent studies showed that two types of processes, deterministic and stochastic, influence the microbial community assembly with varying relative effects depending on geographic scales and strength of environmental gradients [54, 62]. In aquatic ecosystems, cyanobacterial blooms can generate sequential changes both in the overall planktonic structure and in environmental conditions [4]. Future studies should pay more attention to whether the strength of ecological selection and rates of dispersal vary with different cyanobacterial states (e.g., bloom vs. non-bloom) with large sample size, thereby providing directions to better understand the mechanisms governing the balance between deterministic and stochastic processes in plankton succession under changing conditions.

\section{Effect of interspecies interactions on the dynamics of eukaryotic plankton community}

Network analysis can potentially provide deep and unique perspectives on microbial interactions and ecological assembly rules beyond those of simple richness and composition [63]. Here, for the first time, we applied correlation-based network analysis to explore the cooccurrence patterns of abundant and rare eukaryotic plankton communities cross a cyanobacterial bloom event. The resulting plankton network had statistical and structural characteristics similar to those of bacterial ecological networks [28, 64], such as non-randomly connected properties, power-law distribution, and modular structure. The topology of the networks can reflect interactions between microorganisms. For example, the degree value describes the level of connectedness between OTUs, and the betweenness centrality value provides information on how critical an OTU is to the connectedness of a network [45]. Our results showed that rare OTUs had higher degree value but a lower betweenness centrality value than abundant OTUs, but the differences were not significant. According to the generation process of a scale-free topology, keystone nodes are commonly recognized as initiating components in networks [65], which tend to have high degree and low betweenness centrality values [66]. All keystone species in our co-occurrence networks were affiliated to rare taxa with the exception of a conditionally abundant OTU and a conditionally rare and abundant OTU. This suggests that rare species may play an irreplaceable role in maintaining the structure of microbial communities. In a co-occurrence network, the positive interaction is mainly regarded as cooperation [67]. We found that rare taxa showed more positive interactions to non-rare taxa (abundant, moderate, and conditionally rare and abundant taxa) than negative ones. Interactions between microorganisms can support ecosystem function and stability, for instance, rare Symbiodinium taxa can cause a significant increase in the stability of the coralsymbiont community under environmental changes [63]. Similarly, the cooperation between rare and non-rare taxa might contribute to the resilience of the microbial plankton community under a changing environment, because the interaction network of plankton can provide a buffer against the environmental disturbance [49]. On the other hand, species played different roles with respect to modularity. In our study, all 6 module hubs (i.e., nodes highly linked within their own module) and 7 out of 12 connectors (i.e., nodes linking different modules together) belonged to rare OTUs. Shi et al. [68] recently reported that less-abundant taxa can act as important keystone taxa in the rhizosphere networks. The disappearance of these key species may cause modules and networks to break apart [46], and thus rare species can be as important as or more important than the abundant ones in maintaining ecosystem stability.

By comparing the taxonomy of OTU nodes with the network modular structure, we found that the assembly of 
the eukaryotic plankton community was non-randomly determined by taxonomic relatedness, that is, closely related taxa tended to be highly interconnected and clustered together. This non-random pattern was evident in our network modules, because most modules exhibited a distinct temporal variation. Modularity may reflect synergistic relations, competitive interactions, and niche differentiation, leading to non-random patterns of interaction and ultimately contributing to the complexity of ecological networks [69]. The division of the network into modules may shed light on the different groups of nodes performing different functions [70]. For instance, module I was dominated by heterotrophic Ciliophora and Cercozoa, which are important consumers of picophytoplankton and bacteria [71, 72]. Phytoplankton blooms can boost the rate of bacterial growth and production [73]. Therefore, the increase in the abundance of these taxa is most likely derived from the increase in the abundance of their prey or food. Module II, III, and VI were specific to the post-bloom period 2 and were phototroph-dominated (e.g., Chrysophyceae and Cryptophyceae). Cryptophyceae was previously found to be favored by reductions of nutrient loading in shallow lakes [74], providing evidence for the existence of distinct ecological niches over temporal scales in the reservoir ecosystem in tandem with the disappearance of cyanobacterial bloom. In this study, rare species were widely located in all modules as central hubs, linking diverse abundant, moderate, and conditionally rare and abundant taxa. Rare microbes sustain a vast functional gene pool and can indirectly enhance functionality of abundant microbes [20]. Similarly, the interactions between rare taxa and non-rare taxa (abundant, moderate, and conditionally rare and abundant taxa) may affect biogeochemical cycles. For example, ciliates grazing upon picophytoplankton and bacteria likely play an essential role in transferring carbon and nutrients to higher trophic levels [75]. In both the modules I and II, ciliates showed more interactions with Chlorophyta and total (organic) carbon (Fig. S13). Parasitic fungal chytrids are common parasites of phytoplankton (e.g., colonial cyanobacteria and diatoms), which had an important influence on food web dynamics [76]. In the module II, the change in Chytridiomycota abundance was concomitant with the change in the abundance of Diatomea (Fig. S13). Such a synchronous change can affect the transfer of carbon from primary production to higher trophic levels [77]. Moreover, we found total (organic) carbon was significantly related to eukaryotic plankton dynamics (Fig. S13). Through these results, network analysis exhibited potential importance in unraveling the intrinsic mechanisms of interspecific interactions and understanding the roles of community members in ecological processes like carbon dynamics. However, in addition to considering species interactions based on the statistical and structural features of networks, both microbial parasites (e.g., chytrid parasitism on cyanobacteria) and interspecific allelopathy in cyanobacteria based on experiments and models can have large influences on food web structure and function [76, 78], and are essential for a good diagnosis, thereby providing directions for future ecology studies on eukaryotic plankton during cyanobacterial blooms.

\section{Conclusions and implications}

Our results clearly demonstrated that the community composition of rare eukaryotic plankton had a stronger temporal pattern than that of abundant taxa following a cyanobacterial bloom. Due to high levels of dispersal, competition for resources and growth rates, the temporal stability of abundant taxa was greater than that of rare taxa. Both deterministic and stochastic processes simultaneously affected the community assembly of eukaryotic plankton, and random patterns (e.g., ecological drift) were particularly pronounced for the rare taxa. These results expanded our knowledge of the temporal patterns and ecological processes behind changes in the plankton community in a changing environment with emphasize on rare subcommunities. Co-occurrence network analysis further revealed that the synergistic effects between rare and nonrare taxa may play central roles in maintaining the stability of eukaryotic community and ecological function (e.g., carbon transfer or flow within the ecosystem). Additionally, eukaryotic plankton networks followed the same principles as bacterial communities, such as power-law distribution, non-randomly connected properties, module structure and "small-world" properties. In summary, our results provide a new perspective for the ecological significance of rare eukaryotic plankton in aquatic ecosystems, reinforcing recent ideas about microbial interactions among eukaryotic plankton.

Acknowledgements We thank Prof. David M. Wilkinson and Prof. George B. McManus for comments on an earlier version of this paper. This work was supported by the National Key Research and Development Program of China (2017YFA0605203), and the Xiamen Municipal Bureau of Science and Technology (3502Z20172024 and 3502Z20171003), and the National Natural Science Foundation of China (31370471 and 31672312).

Author contributions JY designed the experiments. HC and JRY performed the sample collection and determined the environmental parameters. YX performed the PCR, sequencing, and bioinformatics. YX and JY wrote the first draft of the manuscript, and all authors contributed to and have approved the final manuscript.

\section{Compliance with ethical standards}

Conflict of interest The authors declare that they have no conflict of interest. 
Open Access This article is licensed under a Creative Commons Attribution 4.0 International License, which permits use, sharing, adaptation, distribution and reproduction in any medium or format, as long as you give appropriate credit to the original author(s) and the source, provide a link to the Creative Commons license, and indicate if changes were made. The images or other third party material in this article are included in the article's Creative Commons license, unless indicated otherwise in a credit line to the material. If material is not included in the article's Creative Commons license and your intended use is not permitted by statutory regulation or exceeds the permitted use, you will need to obtain permission directly from the copyright holder. To view a copy of this license, visit http://creativecommons. org/licenses/by/4.0/.

\section{References}

1. Scheffer M, Carpenter SR. Catastrophic regime shifts in ecosystems: linking theory to observation. Trends Ecol Evol. 2003;18:648-56.

2. Moustaka-Gouni M, Vardaka E, Michaloudi E, Kormas KA, Tryfon E, Mihalatou H, et al. Plankton food web structure in a eutrophic polymictic lake with a history in toxic cyanobacterial blooms. Limnol Oceanogr. 2006;51:715-27.

3. Simon M, López-García P, Deschamps P, Moreira D, Restoux G, Bertolino $\mathrm{P}$, et al. Marked seasonality and high spatial variability of protist communities in shallow freshwater systems. ISME J. 2015;9:1941-53.

4. Paerl HW, Otten TG. Harmful cyanobacterial blooms: causes, consequences, and controls. Microb Ecol. 2013;65:995-1010.

5. Filstrup CT, Hillebrand H, Heathcote AJ, Harpole WS, Downing JA. Cyanobacteria dominance influences resource use efficiency and community turnover in phytoplankton and zooplankton communities. Ecol Lett. 2014;17:464-74.

6. Boenigk J, Arndt H. Bacterivory by heterotrophic flagellates: community structure and feeding strategies. Antonie van Leeuwenhoek. 2002;81:465-80.

7. Zubkov MV, Tarran GA. High bacterivory by the smallest phytoplankton in the North Atlantic Ocean. Nature. 2008;455:224-6.

8. Capo E, Debroas D, Arnaud F, Perga ME, Chardon C, Domaizon I. Tracking a century of changes in microbial eukaryotic diversity in lakes driven by nutrient enrichment and climate warming. Environ Microbiol. 2017;19:2873-92.

9. Caron DA, Hutchins DA. The effects of changing climate on microzooplankton grazing and community structure: drivers, predictions and knowledge gaps. J Plankton Res. 2013;35:235-52.

10. Yang JR, Lv H, Isabwea A, Liu LM, Yu XQ, Chen HH, et al. Disturbance-induced phytoplankton regime shifts and recovery of cyanobacteria dominance in two subtropical reservoirs. Water Res. 2017;120:52-63.

11. Logares R, Audic S, Bass D, Bittner L, Boutte C, Christen R, et al. Patterns of rare and abundant marine microbial eukartotes. Curr Biol. 2014;24:813-21.

12. Chen WD, Pan YB, Yang J, Zhang WJ. Patterns and processes in marine microeukaryotic community biogeography from Xiamen coastal waters and intertidal sediments, southeast China. Front Microbiol. 2017;8:1912.

13. Yu Z, Yang J, Zhou J, Yu XQ, Liu LM, Lv H. Water stratification affects the microeukaryotic community in a subtropical deep reservoir. J Eukaryot Microbiol. 2014;61:126-33.

14. Pedrós-Alió C. The rare bacterial biosphere. Annu Rev Mar Sci. 2012:4:449-66.
15. Zhang WJ, Pan YB, Yang J, Chen HH, Holohan B, Vaudrey J, et al. The diversity and biogeography of abundant and rare intertidal marine microeukaryotes explained by environment and dispersal limitation. Environ Microbiol. 2018;20:462-76.

16. Wu WX, Logares R, Huang BQ, Hsieh CH. Abundant and rare picoeukaryotic sub-communities present contrasting patterns in the epipelagic waters of marginal seas in the northwestern Pacific Ocean. Environ Microbiol. 2017;19: $287-300$

17. Liu LM, Liu M, Wilkinson DM, Chen HH, Yu XQ, Yang J. DNA metabarcoding reveals that $200-\mu \mathrm{m}$-size-fractionated filtering is unable to discriminate between planktonic microbial and large eukaryotes. Mol Ecol Resour. 2017;17:991-1002.

18. Debroas D, Hugoni M, Domaizon I. Evidence for an active rare biosphere within freshwater protists community. Mol Ecol. 2015;24:1236-47.

19. Lynch MDJ, Neufeld JD. Ecology and exploration of the rare biosphere. Nat Rev Microbiol. 2015;13:217-29.

20. Jousset A, Bienhold C, Chatzinotas A, Gallien L, Gobet A, Kurm $\mathrm{V}$, et al. Where less may be more: how the rare biosphere pulls ecosystems strings. ISME J. 2017;11:853-62.

21. Shade A, Jones SE, Gaporaso JG, Handeslman J, Knight R, Fierer $\mathrm{N}$, et al. Conditionally rare taxa disproportionately contribute to temporal changes in microbial diversity. mBio. 2014;5: e01371-14.

22. Shade A, Gilbert JA. Temporal patterns of rarity provide a more complete view of microbial diversity. Trends Microbiol. 2015;23:335-40.

23. Liu LM, Yang J, Lv H, Yu XQ, Wilkinson DM, Yang J. Phytoplankton communities exhibit a stronger response to environmental changes than bacterioplankton in three subtropical reservoirs. Environ Sci Technol. 2015;49:10850-8.

24. Xue YY, Yu Z, Chen HH, Yang JR, Liu M, Liu LM, et al. Cyanobacterial bloom significantly boosts hypolimnelic anammox bacterial abundance in a subtropical stratified reservoir. FEMS Microbiol Ecol. 2017;93:fix118.

25. Chen MJ, Chen FZ, Xing P, Li HB, Wu QL. Microbial eukaryotic community in response to Microcystis spp. bloom, as assessed by an enclosure experiment in Lake Taihu, China. FEMS Microbiol Ecol. 2010;74:19-31.

26. Ger KA, Urrutia-Cordero P, Frost PC, Hansson LA, Sarnelle O, Wilson $\mathrm{AE}$, et al. The interaction between cyanobacteria and zooplankton in a more eutrophic world. Harmful Algae. 2016;54:128-44.

27. Hunt DE, Ward CS. A network-based approach to disturbance transmission through microbial interactions. Front Microbiol. 2015;6:1182.

28. Hu AY, Ju F, Hou LY, Li JW, Yang XY, Wang HJ, et al. Strong impact of anthropogenic contamination on the co-occurrence patterns of a riverine microbial community. Environ Microbiol. 2017;19:4993-5009.

29. Jiao S, Chen WM, Wei GH. Biogeography and ecological diversity patterns of rare and abundant bacteria in oil-contaminated soils. Mol Ecol. 2017;26:5305-17.

30. Fuhrman JA, Steele JA. Community structure of marine bacterioplankton: patterns, networks, and relationships to function. Aquat Microb Ecol. 2008;53:69-81.

31. Amaral-Zettler LA, McCliment EA, Ducklow HW, Huse SM. A method for studying protistan diversity using massively parallel sequencing of V9 hypervariable regions of small-subunit ribosomal RNA genes. PLoS ONE. 2009;4:e6372.

32. Magoč T, Salzberg SL. FLASH: fast length adjustment of short reads to improve genome assemblies. Bioinformatics. 2011;27:2957-63. 
33. Caporaso JG, Kuczynski J, Stombaugh J, Bittinger K, Bushman FD, Costello EK, et al. QIIME allows analysis of high-throughput community sequencing data. Nat Methods. 2010;7:335-6.

34. Edgar RC, Haas BJ, Clemente JC, Quince C, Knight R. UCHIME improves sensitivity and speed of chimera detection. Bioinformatics. 2011;27:2194-200.

35. Edgar RC. UPARSE: highly accurate OTU sequences from microbial amplicon reads. Nat Methods. 2013;10:996-8.

36. Wang Q, Garrity GM, Tiedje JM, Cole JR. Naive Bayesian classifier for rapid assignment of rRNA sequences into the new bacterial taxonomy. Appl Environ Microb. 2007;73:5261-7.

37. Dai TJ, Zhang Y, Tang YS, Bai YH, Tao YL, Huang B, et al. Identifying the key taxonomic categories that characterize microbial community diversity using full-scale classification: a case study of microbial communities in the sediments of Hangzhou Bay. FEMS Microbiol Ecol. 2016;92:fiw150.

38. Adl SM, Simpson AGB, Lane CE, Lukeš J, Bass D, Bowser SS, et al. The revised classification of eukaryotes. J Eukaryot Microbiol. 2012;59:429-93.

39. de Vargas C, Audic S, Henry N, Decelle J, Mahé F, Logares R, et al. Eukaryotic plankton diversity in the sunlit ocean. Science. 2015;348:1261605.

40. Preston FW. The commonness, and rarity, of species. Ecology. 1948;29:254-83.

41. Bray JR, Curtis JT. An ordination of the upland forest communities of southern Wisconsin. Ecol Monogr. 1957;27:325-49.

42. Collins SL, Micheli F, Hartt L. A method to determine rates and patterns of variability in ecological communities. Oikos. 2000;91:285-93.

43. Levins R. Evolution in changing environments. Princeton, New Jersey: Princeton University Press; 1968.

44. Cáceres MD, Legendre P. Associations between species and groups of sites: indices and statistical inference. Ecology. 2009;90:3566-74.

45. Ma B, Wang HZ, Dsouza M, Lou J, He Y, Dai ZM, et al. Geographic patterns of co-occurrence network topological features for soil microbiota at continental scale in eastern China. ISME J. 2016;10:1891-901.

46. Guimerà R, Amaral LAN. Functional cartography of complex metabolic networks. Nature. 2005;433:895-900.

47. Erdös P, Rényi A. On the evolution of random graphs. Publ Math Inst Hung Acad Sci. 1960;5:17-61.

48. Sloan WT, Lunn M, Woodcock S, Head IM, Nee S, Curtis TP. Quantifying the roles of immigration and chance in shaping prokaryote community structure. Environ Microbiol. 2006;8: $732-40$.

49. Konopka A, Lindemann S, Fredrickson J. Dynamics in microbial communities: unraveling mechanisms to identify principles. ISME J. 2015;9:1488-95.

50. Nolte V, Pandey RV, Jost S, Medinger R, Ottenwalder B, Boenigk $\mathrm{J}$, et al. Contrasting seasonal niche separation between rare and abundant taxa conceals the extent of protist diversity. Mol Ecol. 2010;19:2908-15.

51. Evans TG, Hofmann GE. Defining the limits of physiological plasticity: how gene expression can assess and predict the consequences of ocean change. Philos Trans R Soc B Biol Sci. 2012;367:1733-45.

52. Caron DA, Countway PD. Hypotheses on the role of the protistan rare biosphere in a changing world. Aquat Microb Ecol. 2009;57:227-38.

53. Yachi S, Loreau M. Biodiversity and ecosystem productivity in a fluctuating environment: the insurance hypothesis. Proc Natl Acad Sci USA. 1999;96:1463-8.

54. Morrison-Whittle P, Goddard MR. Quantifying the relative roles of selective and neutral processes in defining eukaryotic microbial communities. ISME J. 2015;9:2003-11.
55. Bahram M, Kohout $\mathrm{P}$, Anslan S, Harend H, Abarenkov K, Tedersoo L. Stochastic distribution of small soil eukaryotes resulting from high dispersal and drift in a local environment. ISME J. 2016;10:885-96.

56. Liu LM, Yang J, Yu XQ, Chen GJ, Yu Z. Patterns in the composition of microbial communities from a subtropical river: effects of environmental, spatial and temporal factors. PLoS ONE. 2013;8:e81232.

57. Pedrós-Alió C. Marine microbial diversity: can it be determined? Trends Microbiol. 2006;14:257-63.

58. Logares R, Mangot JF, Massana R. Rarity in aquatic microbes: placing protists on the map. Res Microbiol. 2015;166:831-41.

59. Roberto AA, Van Gray JB, Leff LG. Sediment bacteria in an urban stream: spatiotemporal patterns in community composition. Water Res. 2018;134:353-69.

60. Magurran AE, Henderson PA. Explaining the excess of rare species in natural species abundance distributions. Nature. 2003;422:714-6.

61. Burns AR, Stephens WZ, Stagaman K, Wong S, Rawls JF, Guillemin K, et al. Contribution of neutral processes to the assembly of gut microbial communities in the zebrafish over host development. ISME J. 2016;10:655-64.

62. Hanson CA, Fuhrman JA, Horner-Devine MC, Martiny JB. Beyond biogeographic patterns: processes shaping the microbial landscape. Nat Rev Microbiol. 2012;10:497-506.

63. Ziegler M, Eguíluz VM, Duarte CM, Voolstra CR. Rare symbionts may contribute to the resilience of coral-algal assemblages. ISME J. 2018;12:161-72.

64. Zhao DY, Shen F, Zeng J, Huang R, Yu ZB, Wu QL. Network analysis reveals seasonal variation of co-occurrence correlations between Cyanobacteria and other bacterioplankton. Sci Total Environ. 2016;573:817-25.

65. Barabási AL. Scale-free networks: a decade and beyond. Science. 2009;325:412-3.

66. Berry D, Widder S. Deciphering microbial interactions and detecting keystone species with co-occurrence networks. Front Microbiol. 2014;5:219.

67. Ju F, Xia Y, Gou F, Wang Z, Zhang T. Taxonomic relatedness shapes bacterial assembly in activated sludge of gloablly distributed wastewater treatment plantis. Environ Microbiol. 2014;16:2421-32.

68. Shi SJ, Nuccio EE, Shi ZJ, He ZL, Zhou JZ, Firestone MK. The interconnected rhizosphere: high network complexity dominates rhizosphere assemblages. Ecol Lett. 2016;19:926-36.

69. Olesen JM, Bascompte J, Dupont YL, Jordano P. The modularity of pollination networks. Proc Natl Acad Sci USA. 2007;104:19891-6.

70. Xiong W, Jousset A, Guo S, Karlsson I, Zhao QY, Wu HS, et al. Soil protist communities form a dynamic hub in the soil microbiome. ISME J. 2018;12:634-8.

71. Sherr EB, Sherr BF. Significance of predation by protists in aquatic microbial food webs. Antonie van Leeuwenhoek 2002;81:293-308.

72. Cavalier-Smith T, Chao EEY. Phylogeny and classification of phylum Cercozoa (Protozoa). Protist. 2003;154:341-58.

73. Bird DF, Karl DM. Uncoupling of bacteria and phytoplankton during the austral spring bloom in Gerlache Strait, Antarctic Peninsula. Aquat Microb Ecol. 1999;19:13-27.

74. Jeppesen E, Søndergaard M, Meerhoff M, Lauridsen TL, Jensen JP. Shallow lake restoration by nutrient loading reduction - some recent findings and challenges ahead. Hydrobiologia. 2007;584:239-52.

75. Worden AZ, Follows MJ, Giovannoni SJ, Wilken S, Zimmerman AE, Keeling PJ. Rethinking the marine carbon cycle: factoring in the multifarious lifestyles of microbes. Science. 2015;347:1257594.

76. Haraldsson M, Gerphagnon M, Bazin P, Colombet J, Tecchio S, Sime-Ngando $\mathrm{T}$, et al. Microbial parasites make cyanobacteria 
blooms less of a trophic dead end than commonly assumed. ISME J. 2018;12:1008-20.

77. Kagami M, Helmsing NR, van Donk E. Parasitic chytrids could promote copepod survival by mediating material transfer from inedible diatoms. Hydrobiologia. 2011;659:49-54.
78. Rzymski P, Poniedziałek B, Kokociński M, Jurczak T, Lipski D, Wiktorowicz K. Interspecific allelopathy in cyanobacteria: cylindrospermopsin and Cylindrospermopsis raciborskii effect on the growth and metabolism of Microcystis aeruginosa. Harmful Algae. 2014;35:1-8. 